\title{
Effects of cholesterol and unsaturated DOPC lipid on chain packing of saturated gel-phase DPPC bilayers
}

\author{
Thalia T. Mills ${ }^{1}$, Juyang Huang ${ }^{2}$, Gerald W. Feigenson ${ }^{3}$ and John F. Nagle ${ }^{1}$ \\ ${ }^{1}$ Department of Physics, Carnegie Mellon University, Pittsburgh, Pensylvania 15213, U.S.A. \\ ${ }^{2}$ Department of Physics, Texas Tech University, Lubbock, Texas 79409, U.S.A. \\ ${ }^{3}$ Field of Biophysics, Cornell University, Ithaca, New York 14853, U.S.A.
}

\begin{abstract}
Wide angle x-ray scattering (WAXS) from oriented lipid multilayers was used to study the effect of adding cholesterol (Chol) or 1,2-dioleoyl-sn-glycero-3-phosphocholine (DOPC) to gel-phase 1,2-dipalmitoyl-sn-glycero-3-phosphocholine (DPPC) bilayers. Small quantities $(X<0.10$ mole fraction) of both molecules disrupt the tight packing of tilted chains of pure gel-phase DPPC, forming a more disordered, untilted phase. The addition of larger quantities of DOPC causes the sample to phase-separate into a gel phase, characterized by a narrow WAXS peak, and liquid disordered phase, characterized by wide, diffuse WAXS scattering. In contrast, two WAXS peaks indicative of two coexisting phases were not observed in Chol/DPPC mixtures $\left(X_{\mathrm{Chol}}=0.07\right.$ to 0.40$)$. Instead, Chol caused a gradual increase in the width of the WAXS peak, consistent with a gradual change from a more gel-like to a more liquid-like state rather than passing through a region of two phase coexistence. Our WAXS data include a huge amount of information. A new method of analysis suggests that WAXS data may provide definitive results relating to the disagreements between previously published phase diagrams for Chol/DPPC.
\end{abstract}

Key words: Cholesterol — Liquid ordered — Lipid bilayers — Chain packing — Phase diagram

Abbreviations: WAXS, wide angle x-ray scattering; Chol, cholesterol; DOPC, 1,2-dioleoyl-sn-glycero3-phosphocholine; DPPC, 1,2-dipalmitoyl-sn-glycero-3-phosphocholine; $\mathrm{L}_{\mathrm{O}}$, liquid ordered; $\mathrm{L}_{\mathrm{d}}$, liquid disordered; $X$, mole fraction; $\mathrm{L}_{\beta}$, tilted gel; $\mathrm{P}_{\beta}$, ripple phase gel; $T_{\mathrm{P}}$ pretransition temperature; $T_{\mathrm{m}}$, main transition temperature; DMPC, 1,2-dimyristoyl-sn-glycero-3-phosphocholine; GUV, giant unilamellar vesicle; NMR, nuclear magnetic resonance; DSC, differential scanning calorimetry; $D$, lamellar repeat; $d$, WAXS spacing; LAXS/SAXS, low/small angle x-ray scattering; MLV, multilamellar vesicle; HWHM, half width at half maximum; CHESS, Cornell high energy synchrotron source; $S$, sample-to-detector; $\alpha$, incident angle of $\mathrm{x}$-ray beam on the oriented samples; CCD, charge coupled device; $q$, magnitude of scattering wavevector; $q_{\mathrm{r}}$ and $q_{\mathrm{z}}$, horizontal and vertical components of the scattering wavevector; $\varphi$, angle on the detector between $q_{\mathrm{r}}$ and $q_{\mathrm{z}} ; \beta$, lipid chain tilt angle with respect to normal.

\section{Introduction}

Cholesterol (Chol) has an important role in maintaining a cell membrane that has high fluidity (fast lateral diffusion) but is relatively rigid and impermeable (Lindblom

Correspondence to: John F. Nagle, Department of Physics, Cegie Mellon University, 5000 Forbes Avenue, Wean Hall, Rm. 7322, Pittsburgh, Pensylvania 15213, U.S.A.

E-mail: nagle@cmu.edu et al. 1981; Yeagle 1985; Finegold 1993). These seemingly conflicting properties result from two of Chol's rather unique properties: i) its ability to orientationally order chains of fluid phase-lipids (Sankaram and Thompson 1990; Huang and Feigenson 1999), which leads to increased bilayer thickness (Gallová et al. 2004; Kučerka et al. 2008), smaller partial molecular volume (Greenwood et al. 2006) and area (Edholm and Nagle 2005), and decreased permeability (Mathai et al. 2008); and ii) its ability to cause positional/translational disordering of gel-phase lipids while 
still maintaining a high degree of orientational ordering (Ipsen et al. 1987). The high Chol content state characterized by low positional order and fast diffusion but high orientational/conformational order is termed the "liquid ordered $\left(\mathrm{L}_{\mathrm{o}}\right)$ phase."

Mixtures of dipalmitoylphosphatidylcholine (DPPC) and Chol are perhaps the most studied system for characterizing the change from the well-ordered gel phase in pure DPPC to the $\mathrm{L}_{\mathrm{o}}$ phase with increasing Chol content. Pure DPPC is in a tightly packed arrangement of tilted chains $\left(\mathrm{L}_{\beta}\right.$, phase) below its pretransition temperature $\left(T_{\mathrm{P}}=34^{\circ} \mathrm{C}\right.$ ) (Tardieu et al. 1973; Janiak et al. 1976; Tristram-Nagle et al. 1993). It is also generally agreed that the addition of sufficient amounts of Chol results in a state that is conventionally called the $\mathrm{L}_{\mathrm{o}}$ phase. However, there is disagreement over how the $\mathrm{L}_{\mathrm{o}}$ phase develops thermodynamically as a function of Chol concentration.

The much-cited Vist and Davis (1990) phase diagram proposes gel and $\mathrm{L}_{\mathrm{O}}$ phase coexistence for mole fraction of Chol $\left(X_{\mathrm{Chol}}\right)$ from 0.07 to 0.22 when the temperature is below the main transition temperature which occurs at $T_{\mathrm{m}}=37^{\circ} \mathrm{C}$ for deuterated DPPC (the ripple phase was ignored in that phase diagram). That phase diagram also has the coexistence of liquid disordered $\left(\mathrm{L}_{\mathrm{d}}\right)$ and $\mathrm{L}_{\mathrm{o}}$ phases at higher temperatures. Similar phase diagrams have been published for other binary sterol/lipid mixtures, such as Chol/DMPC (Almeida et al. 1993) and ergosterol/DPPC (Hsueh et al. 2005). Different techniques report different phase boundaries; for instance, an early volumetric study presented evidence for phase coexistence for $X_{\mathrm{Chol}}=0.20$ to 0.29 for $T<T_{\mathrm{m}}\left(41^{\circ} \mathrm{C}\right.$ for undeuterated DPPC) (Melchior et al. 1980). Some methods report no phase coexistence, most notably fluorescence microscopy images of giant unilamellar vesicles (GUVs), which are uniform at all temperatures for Chol/DPPC and other binary Chol/phospholipid mixtures (Feigenson and Buboltz 2001; Veatch and Keller 2005). The microscopy results suggest that any spatial variations in composition could only involve domains less than one micron in diameter.

Differences in time and distance scales probed by different techniques (Bloom and Thewalt 1995) do not fully account for discrepancies in the literature. For instance, reports of phase coexistence in the Chol/DPPC system using nuclear magnetic resonance (NMR) and/or differential scanning calorimetry (DSC) strongly disagree over the location of phase boundaries and nature of the phases (Vist and Davis 1990; Huang et al. 1993; McMullen and McElhaney 1995). Whether nanoscale heterogeneities, observable by spectroscopic methods but not by optical microscopy, constitute true thermodynamic phases has also been questioned (Huang et al. 1993; Feigenson and Buboltz 2001; Heerklotz and Tsamaloukas 2006; McConnell and Radhakrishnan 2006; Veatch et al. 2006; Krivanek et al. 2008).
Most of the X-ray diffraction literature concerning phase behavior of Chol/DPPC mixtures focuses on the low temperature region, and again there are differences. There are some reports of gel/fluid phase coexistence based on the observation of two lamellar repeat $(D)$ spacings in low angle x-ray scattering (LAXS, often called SAXS) (Rand et al. 1980; Meyer et al. 1997) or two wide angle $\mathrm{x}$-ray scattering (WAXS) spacings $d$ (Engelman and Rothman 1972), but these reports are not in agreement with the location of phase boundaries $\left(X_{\mathrm{Chol}}=\right.$ 0.03 to 0.10 in Rand et al. (1980), Meyer et al. (1997); and $X_{\text {Chol }}=0.20$ to 0.33 in Engelman and Rothman (1972)). In other reports, no x-ray evidence was found for gel/fluid phase separation in Chol/DPPC (Ladbrooke et al. 1968; Karmakar et al. 2006) and Chol/DMPC (Hui and He 1983; Finean 1990). There are also indirect $\mathrm{x}$-ray reports of high temperature $\mathrm{L}_{\mathrm{d}} /$ $\mathrm{L}_{\mathrm{O}}$ coexistence in Chol/DPPC mixtures (Richter et al. 2001; Petrache et al. 2005; Chen et al. 2007), but two clearly resolved lamellar repeat or WAXS spacings were not observed in these studies. The majority of $\mathrm{x}$-ray measurements have been carried out on unoriented multilamellar vesicle (MLV) samples, but in recent small angle $\mathrm{x}$-ray studies of Chol/DPPC oriented samples (Karmakar and Raghunathan 2003), experiments at $98 \%$ relative humidity revealed a new gel phase and novel phase diagram. However, this new phase was not observed in a subsequent study by the same group conducted at higher hydration (Karmakar et al. 2006), as only single lamellar repeats were observed over a range of Chol concentrations.

As discussed in a previous publication (Mills et al. 2008b), LAXS can be inadequate to detect the presence/absence of phase coexistence because the absence of two lamellar repeats does not necessarily imply a single phase (Rand et al. 1980). Because it is capable of detecting phase separation within single bilayers, WAXS has an advantage over lamellar repeat scattering. Hui and $\mathrm{He}$ (1983) published one of the most extensive wide angle scattering studies of binary Chol/lipid mixtures in isotropic MLV samples. For $X_{\mathrm{Chol}}=$ 0.075 to 0.45 in DMPC and temperatures ranging from 2 to $40^{\circ} \mathrm{C}$, Hui and $\mathrm{He}$ (1983) observed only a single broad wide angle $\mathrm{x}$-ray and electron diffraction band. Because of the failure to observe the coexistence of a narrow WAXS peak (characteristic of gel) and broader WAXS peak (characteristic of fluid), this study concluded that the gradual change in the wide angle pattern "indicates that there is a continuous change in structure as the Chol content increases, rather than a coexistence of two phase-separated regions in the specimen."

Oriented samples can give information about orientational ordering not available from unoriented MLV samples, as has been shown for fluid phases (Levine and Wilkins 1971; Mills et al. 2008a) but also for gel phase packing and orientation (Smith et al. 1990; Tristram-Nagle et al. 1993). Therefore, a re-examination of the Chol/DPPC system focusing on WAXS data from oriented multilayer samples is of value not 
only for examining the question of phase coexistence, but also in better characterizing the structure (positional and orientational ordering) in these mixtures.

Although the high temperature $\left(T>T_{\mathrm{m}}\right)$ portion of the Vist and Davis phase diagram has received great attention because of the possible relevance of $\mathrm{L}_{\mathrm{d}} / \mathrm{L}_{\mathrm{o}}$ phase coexistence to cell membrane rafts, Vist and Davis's proposed phase diagram is strongly based on data collected in the low temperature $\left(T<T_{\mathrm{m}}\right)$ region. The low temperature behavior of these mixtures (the focus of this report) is of interest because of the conflict between Hui and He's result and phase diagrams which predict gel/ $\mathrm{L}_{\mathrm{o}}$ coexistence and also because of the large structural changes in gel-phase chain packing caused by Chol, which are directly measurable with WAXS.

While a single miscible fluid phase with smoothly changing properties is thermodynamically feasible, the transition from a solid with crystalline long range order to a disordered fluid most commonly occurs via a first order phase transition; therefore, a gradual/continuous change from pure DPPC gel to the $\mathrm{L}_{\mathrm{o}}$ phase at high Chol concentrations, as suggested from some reports (Hui and He 1983; Feigenson and Buboltz 2001), might seem impossible. However, the gel phase does not have three dimensional crystalline order. The gel phase involves order in two dimensions, but long range order cannot exist in two dimensional systems (Mermin and Wagner 1966; Mermin 1968), so even though the positional order, as measured by the (2,0) peak (Sun et al. 1994), extends over at least $290 \mathrm{~nm}$, this conceptual argument for a first order transition is flawed.

For comparison, we first present WAXS results for the less controversial system DOPC/DPPC. When DOPC is added to DPPC, the mixture separates into DPPC-rich gel phase domains and DOPC-rich fluid phase domains, as has been directly observed by fluorescence microscopy (Veatch and Keller 2003; Li and Cheng 2006). Chol or DOPC can both be considered "impurities" which disrupt the tight gel-phase packing of DPPC; however, the structural changes to pure DPPC gel phase are very different for these two impurities as we show in this paper.

In a previous study (Mills et al. 2008b), we focused on using WAXS to examine $\mathrm{L}_{\mathrm{d}} / \mathrm{L}_{\mathrm{o}}$ phase coexistence in ternary mixtures containing DOPC, DPPC, and Chol. Phase behavior of Chol/DPPC and DOPC/DPPC binary mixtures are also important with regard to constructing a complete phase diagram of this DOPC/DPPC/Chol ternary system because the binary systems form two sides of the triangle commonly used to represent ternary systems.

\section{Materials and Methods}

For all sample preparations (oriented and unoriented), mixtures of DPPC and DOPC (Avanti Polar Lipids,
Alabaster, AL), and Chol (Avanti Polar Lipids; Nu Chek Prep, Elysian, MN) were prepared from stock solutions in high-performance liquid chromatography grade chloroform (Fisher Scientific, Pittsburgh, PA). Purity $>99.5 \%$ was confirmed by thin layer chromatography. The molar concentrations of the phospholipid stock solutions were determined by phosphate assay (Kingsley and Feigenson 1979).

Oriented samples consisting of $\sim 1800$ bilayers $(10 \mu \mathrm{m}$ thick) were prepared using the rock and roll technique (Tristram-Nagle 2007). For oriented samples, $4 \mathrm{mg}$ total of lipid was dissolved in $200 \mu$ lorganic solvent (chloroform/ methanol in ratios varying from $3: 1$ to $20: 1$ ) and then deposited on a $30 \times 15 \times 1 \mathrm{~mm}$ silicon wafer. After thorough drying, the samples were trimmed to a $5 \mathrm{~mm}$ strip occupying only the center of the $15-\mathrm{mm}$ wide silicon wafer. The samples were annealed in a high-humidity chamber at $50^{\circ} \mathrm{C}$ for typically $4-12 \mathrm{~h}$, and then allowed to slowly cool to room temperature over $\sim 4-8 \mathrm{~h}$. According to previously reported rocking curves (Mills et al. 2008a), this method produced samples with mosaicities less than $0.03^{\circ}$ half width at half maximum (HWHM).

As has been described elsewhere, the sample chamber (Kučerka et al. 2005) provided a carefully regulated thermal environment that allowed achievement of full hydration from the vapor for oriented samples. However, it is a challenge to reach full hydration without depositing a layer of excess water on top of the oriented lipid stack ("flooding"). Water scattering in the WAXS region can obscure the lipid scattering (Mills et al. 2008a). To avoid flooding, we typically took WAXS data with $D=2-5 \AA$ below the fully hydrated value that was determined from experiments on unoriented MLVs immersed in water in capillaries.

X-ray data were collected at both the Cornell high energy synchrotron source (CHESS) and at a rotating anode source, and oriented data presented here are labeled "synchrotron" or "rotating anode." The synchrotron setup at the CHESS D-1 station has been previously described (Mills et al. 2008a). Scattering as a function of detector pixels was converted to intensity as a function of the scattering wavevector, $q=4 \pi \sin \theta / \lambda$, where $2 \theta$ is the total angle through which the $\mathrm{x}$-rays scatter and $\lambda$ is the $\mathrm{x}$-ray wavelength. Further data collection and analysis procedures, which were essentially the same for the synchrotron and rotating anode, have also been described in detail previously (Mills et al. 2008a).

Rotating anode $\mathrm{x}$-ray measurements on oriented samples at Carnegie Mellon used a $1 \times 1 \mathrm{~mm}$ beam from the $\mathrm{Cu}$ $\mathrm{K} \alpha$ line $(\lambda=1.5418 \AA)$ of a Rigaku RUH3R rotating anode (Woodlands, TX) equipped with a Xenocs FOX2D focusing collimator. Scans were collected using a $1 \mathrm{~K} \times 1 \mathrm{~K}$ Rigaku Mercury CCD detector with a pixel size of $68.0 \mu \mathrm{m} /$ pixel. 
Sample-to-detector $(S)$ distances of 96.64 and $97.7 \mathrm{~mm}$ were calibrated with silver behenate $(D=58.367 \AA)$. Temperature was controlled with a Julabo controller (Allentown, PA) and monitored with a Cole-Parmer thermistor thermometer (Vernon Hills, IL). As for the synchrotron setup, the incident angle $\alpha$ of the beam on the flat samples was adjustable to $0.01^{\circ}$ precision by a rotation stage inside the chamber. Data were collected at $\alpha=0.1^{\circ}$, and mylar window scattering was eliminated from the images by subtracting a "light background" in which the sample was rotated to $-\alpha$. During the long 10-20 min WAXS exposures, an extension on a Mo beamstop blocked the strong lamellar orders. This extension was moved in order to obtain $D$ spacing measurements, which were short exposures ( 30 s) collected while continuously rotating the oriented sample from $\alpha=-3$ to $7^{\circ}$.

MLVs in excess water were prepared in several ways and measured with different setups. Preparation and setup conditions are referred to in the text as MLV 1, MLV 2 and MLV 3 and are described as follows:

- MLV 1: MLVs were made using the "low temperature trapping" method. This method, which helps prevent artifactual Chol precipitation, has been described in detail elsewhere (Huang et al. 1999). Briefly, $1.5 \mathrm{mg}$ lipid was lyophilized from chloroform, while controlling the temperature so that the chloroform remained solid. After removal of bulk solvent, the powders were kept at $-20^{\circ} \mathrm{C}$ under vacuum for about $12 \mathrm{~h}$. Samples were hydrated with excess water, vortexed, and then loaded into $1 \mathrm{~mm}$ glass capillaries (Charles Supper Co., Cambridge, MA) and centrifuged at $5000 \times g$ for 15 min to produce a dense pellet. Capillaries were sealed by paraffin wax under argon gas. $\mathrm{x}$-ray diffraction measurements at room temperature $\left(T=24^{\circ} \mathrm{C}\right)$ were performed at the A-1 beamline at the macromolecular diffraction facility at the CHESS (MacCHESS) as described previously (Huang et al. 1999). An $S$ distance of $295 \mathrm{~mm}$ was used, which allowed the low and wide angle diffraction data $\left(q=0.06 \AA^{-1}\right.$ to $\left.1.65 \AA^{-1}\right)$ to be collected on the same image. Exposure times were $10-100 \mathrm{~s}$.

- MLV 2: WAXS data from one DOPC/DPPC mixture is presented in Figure 2 using the sample preparation protocol and x-ray setup previously described (Zhao et al. 2007). Briefly, $\sim 5 \mathrm{mg}$ lipid was lyophilized at $-10^{\circ} \mathrm{C}$ from a cyclohexane/methanol mixture (containing $<10 \%$ methanol) for $1-2 \mathrm{~h}$. Then $6 \mu \mathrm{l}$ of water were added to the lipid, yielding a final concentration of $\sim 1: 1$ water : lipid. Samples were then cycled 3 times between $-196^{\circ} \mathrm{C}$ (liquid nitrogen temperature) and $75^{\circ} \mathrm{C}$, mechanically mixing during each cycle. $\mathrm{x}$-ray measurements at Cornell used a Ni-filtered Cu K $\alpha$ line $(\lambda=1.5418 \AA)$ of a Rigaku RU3HR rotating anode Woodlands $\mathrm{x}$-ray source operated at $38 \mathrm{kV}$ and $50 \mathrm{~mA}$. $\mathrm{x}$-rays were focused using orthogonal Franks mirrors, and tantalum slits trimmed the beam to $1 \times 1 \mathrm{~mm}$. WAXS images were collected on a homebuilt $1 \mathrm{~K} \times 1 \mathrm{~K}$ pixel CCD detector (Tate et al. 1997). The $S$ distance of $95.7 \mathrm{~mm}$ was calibrated with p-bromo-benzoic acid. Exposure time was $40 \mathrm{~min}$. Sample temperature was controlled with a water-cooled Peltier controller (Melcor Inc., Trenton, NJ). The beamline including sample chamber were kept evacuated during measurements to reduce air scattering background.

- MLV 3: Lamellar repeats for DOPC/DPPC and Chol/ DPPC mixtures have been previously reported (see Supplement Data S5 for Mills et al. 2008a). Briefly, these MLVs were prepared by adding water to a dry lipid film for a final water : lipid mass ratio of $5: 1$ to $10: 1$ and then carrying the lipid through three freeze/thaw cycles (Mills et al. 2008a). The $\mathrm{x}$-ray measurements were performed using the same rotating anode and detector as described for MLV 2, but with a longer $S$ distance of 385.8 or $306.7 \mathrm{~mm}$, as calibrated with silver behenate.

\section{Results and Discussion}

\section{DOPC removes DPPC chain tilt and induces gel/fluid phase} coexistence

Fig. 1A shows oriented WAXS data from pure DPPC at $25^{\circ} \mathrm{C}$. As has been known for many years (Tardieu et al. 1973; McIntosh 1978; Tristram-Nagle et al. 1993) DPPC has an equatorial $(2,0)$ peak and the $(1,1)$ peak lifted off from the equator. This is often designated the $\mathrm{L}_{\beta}$, phase, although it is more precisely designated the $L_{\beta}$ 'I phase (Smith et al. 1990) where the final I subscript indicates that the chains are tilted toward nearest neighbors. The satellite peaks (Sun et al. 1994) on the $(1,1)$ Bragg rod may also be seen in Fig. 1A. The $q_{\mathrm{r}}$ width of the $(2,0)$ peak in Fig. $1 \mathrm{~A}$ is resolution limited, so it shows experimental resolution for this study.

In contrast, scattering from pure $\mathrm{L}_{\mathrm{d}}$ phase $\mathrm{DOPC}$ is very broad as shown in Fig. 1C. The breadth in the $q_{\mathrm{r}}$ direction indicates positional disorder and the breadth in the $\varphi$ direction indicates orientational disorder (Mills et al. 2008a). Fig. 1B shows data for equimolar DOPC/DPPC. There is clearly a broad diffuse band that is instrumentally resolved. We assign this to an $L_{d}$ phase that is similar to the $L_{d}$ phase of pure DOPC. There is also a prominent, relatively sharp peak that we ascribe to a gel phase. This peak is broader than the gel phase peaks in Fig. 1A. The fact that this peak is instrumentally resolved means that there is less positional order in this DOPC/DPPC gel phase than in the pure DPPC gel phase.

The gel phase in the DOPC/DPPC mixture is even more strikingly different from the pure DPPC gel phase 


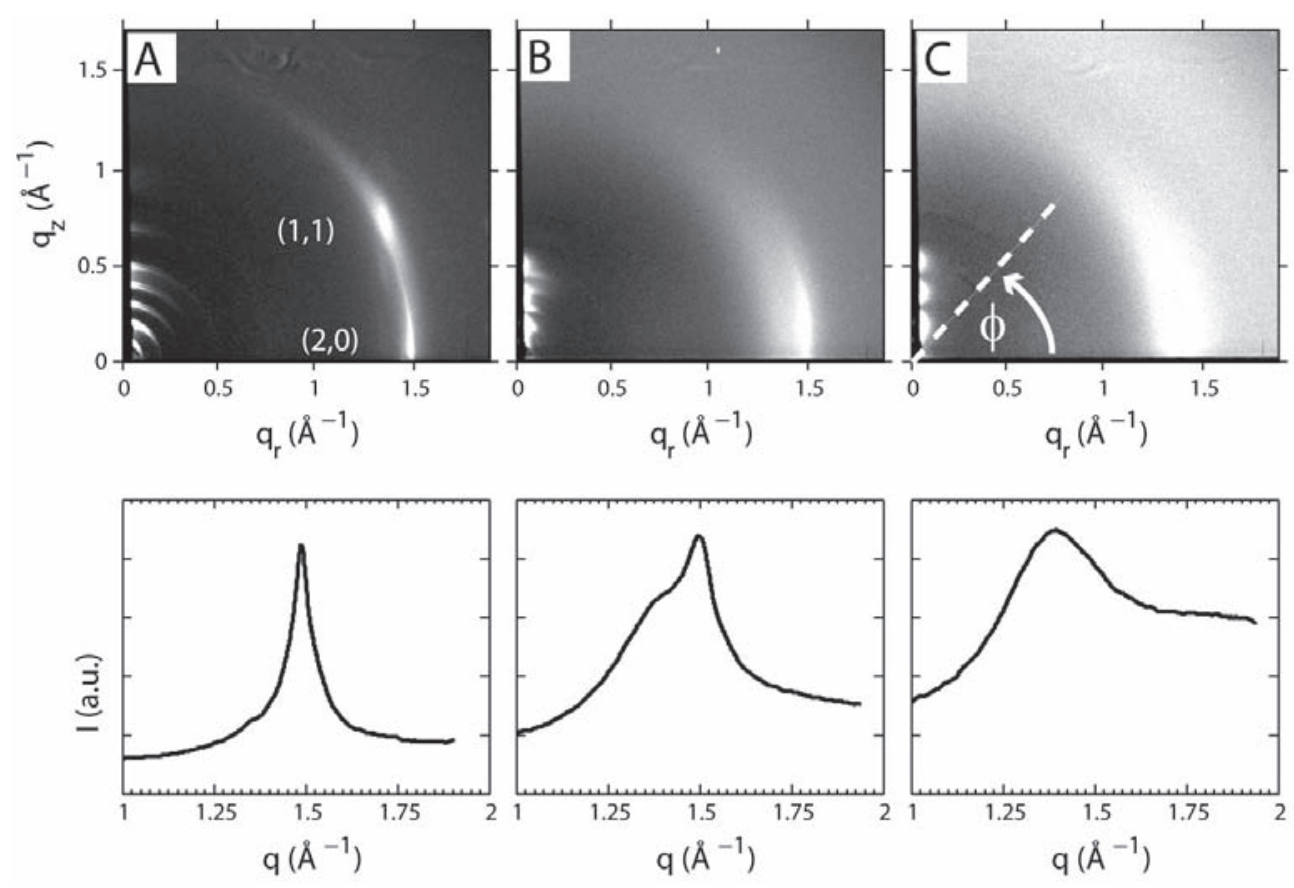

Figure 1. Linear grayscale CCD images show high intensity with white pixels. The spatial axes have been approximately converted to scattering vector components $q_{\mathrm{z}}$ along the normal to the bilayers and $q_{\mathrm{r}}$ in the plane of the bilayers. The scattering near $q_{\mathrm{r}}=0$ is LAXS lamellar scattering that is mostly absorbed by a beamstop extension. WAXS scattering is concentrated near $q \sim 1.5 \AA^{-1}$. All three images were taken with the same setup at CHESS. A. DPPC in the $\mathrm{L}_{\beta}$, phase at $25^{\circ} \mathrm{C}(D=63.4 \AA$; same as fully hydrated data obtained with setup MLV 3). B. Equimolar DOPC/DPPC at $25^{\circ} \mathrm{C}\left(D=62.8 \AA\right.$; compared with $D=63.2 \AA$ for MLV 3). C. DOPC in the $\mathrm{L}_{\mathrm{d}}$ phase at $25^{\circ} \mathrm{C}(D=$ $60.9 \AA$ compared with $63 \AA$ for MLVs). The bottom row shows the corresponding $I(q)$ plots integrated over a $\varphi$ range of 5-15 . Data collection and processing are described in detail in a previous paper (Mills et al. 2008a). All synchrotron data.

as there is only one relatively sharp component centered on the equator in Fig. 1B. This suggests that it is an $\mathrm{L}_{\beta}$ phase with an average chain tilt of zero. These differences compared to the pure DPPC gel phase in Fig. 1A are proof that it is not pure DPPC and that whatever concentration of DOPC is in the gel phase is sufficient to remove the tilt of the hydrocarbon chains. It may be noted that this disagrees with a recent polarization microscopy study (Li and Cheng 2006) that concluded that the gel phase coexisting with the fluid phase in DOPC/DPPC mixtures was either $\mathrm{L}_{\beta}$, or $\mathrm{P}_{\beta}$, depending on composition and temperature. However, our DOPC/DPPC gel phases have very clear WAXS signatures, and we only saw evidence of an untilted $\left(\mathrm{L}_{\beta}\right.$-like) phase.

Fig. 2 shows unoriented WAXS data for equimolar DOPC/DPPC at $25^{\circ} \mathrm{C}$. Narrow (gel) and broad (fluid) peaks are clearly discernable in the data for this system. In contrast, in another study (Chen et al. 2007) the broad fluid-phase WAXS peak was not observed for this same lipid mixture. The data shown in Fig. 2 are a best-case scenario for collecting high lipid signal to noise (minimal $1: 1$ water : lipid mass ratio, entire beamline under vacuum).
Usually, in order to ensure full hydration and mixing of unoriented samples, we make samples with mass ratios $5: 1$ to $10: 1$ water : lipid, closer to the $4: 1$ ratio used in the other study (Chen et al. 2007). Under these conditions, the broad WAXS fluid peak can be obscured because of increase in the high- $q$ water scattering near $q=2.0 \AA^{-1}$. Oriented samples avoid this excess water problem and, more importantly, they also give additional information about the chain orientation/tilt.

Fig. 3 shows that the structure of $0.10 / 0.90$ DOPC/ DPPC is sensitive to hydration/equilibration. After first loading the sample into the hydration chamber, the chains are tilted, as in pure gel phase DPPC (Fig. 3A). The equatorial and tilted peaks are still observable after $3.5 \mathrm{~h}$. (Fig. $3 \mathrm{~B})$, but only an equatorial peak is observable after the sample equilibrated overnight at high humidity (Fig. 3C). The transformation is not simply hydration-dependent, as the data shown in Fig. 3B,C have nearly equal $D$ spacings, showing that equilibration time is also a factor. Furthermore, this transformation is reversible by reducing the relative humidity as seen in Fig. 3D. Samples of 0.15/0.85 and 0.05/0.95 DOPC/DPPC mixtures exhibited similar 

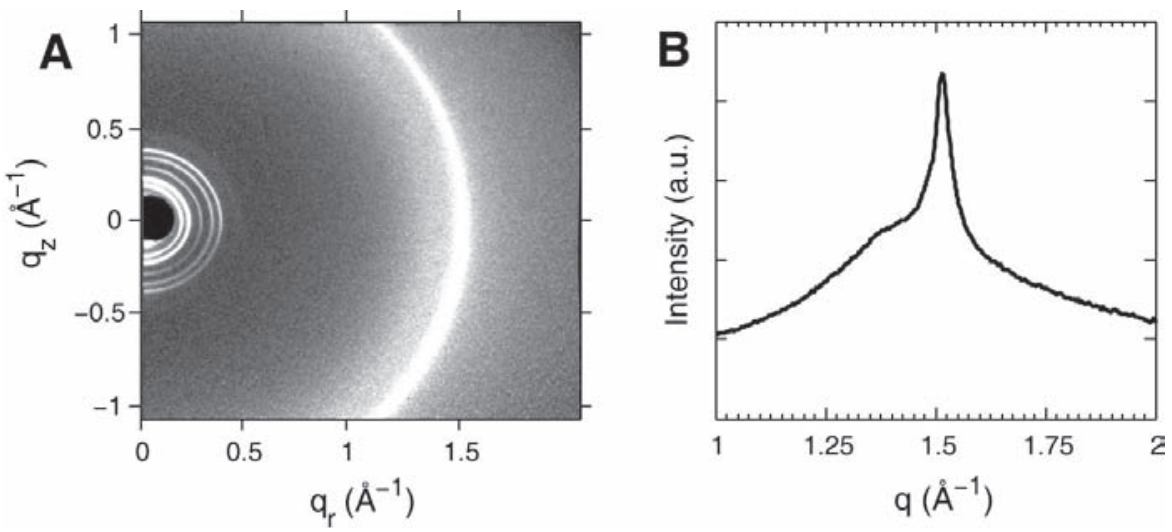

Figure 2. A. Unoriented WAXS data for a fully hydrated sample of equimolar DOPC/DPPC prepared from a lyophilized powder with a 1:1 water : lipid mass ratio. Data collected with setup MLV 2 as described in Materials and Methods. B. Corresponding I $(q)$ plot integrated over the entire image.
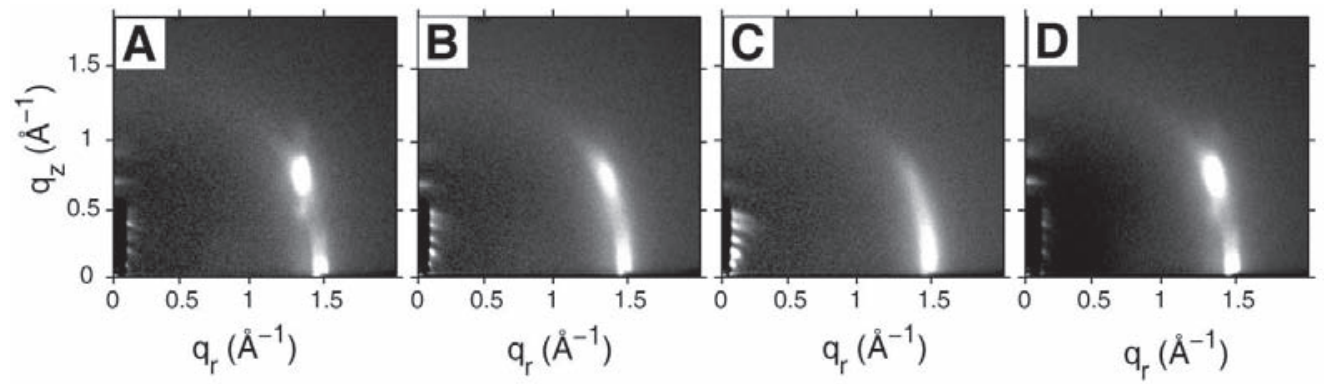

Figure 3. Time series of oriented WAXS data for 0.10/0.90 DOPC/DPPC after loading the sample into the chamber and beginning hydration at time $t=0$ : A. $t=30 \mathrm{~min}$., $D=60.9 \AA \AA$; B. $T=3.5 \mathrm{~h}, D=63.8 \AA ̊$; C. $T=18$ h, $D=63.5 \AA$; D. after blowing a weak stream of dry helium over the sample, $D=61.0 \AA$. All rotating anode data.

behavior. However, the time for disappearance of the tilted peak occurred faster for the larger DOPC concentrations (less than $3 \mathrm{~h}$ for the $0.15 / 0.85$ DOPC/DPPC sample). Dependence of gel-phase structure on hydration conditions has been studied in great detail for pure DMPC (Smith et al. 1990; Tristram-Nagle et al. 2002), where dehydration reduces the chain tilt as well as changing the azimuthal in-plane direction of the tilt. In contrast, in Fig. 3 dehydration increases the chain tilt and there is no evidence for change in tilt direction.

Based on previous reports (Lentz et al. 1976; Veatch and Keller 2003), 0.10/0.90 DOPC/DPPC at $25^{\circ} \mathrm{C}$ should be just within the gel-fluid coexistence region. If so, the gel phase observed for this mixture should have the same properties as the gel phase in the equimolar mixture. For instance, Fig. 3A, if it represented the hydrated, equilibrium structure, would be inconsistent with Fig. $1 \mathrm{~B}$. These results show that the gel phase bilayer structure can be strongly dependent on equilibration time and hydration, and so it is important to use a chamber capable of retaining high humidity over a long time if oriented sample data are to be compared with other measurements taken in excess water conditions.

Even smaller amounts of DOPC also remove the chain tilt from DPPC bilayers, as we have verified for 0.05/0.95 DOPC/DPPC (data not shown). Presumably, there is some smaller DOPC concentration, call it $X_{0 \text { tilt }}$, below which there is a chain tilt. It is interesting to compare to the effect of DOPC on DSPC lipid which also forms the $\mathrm{L}_{\beta}$, phase. According to previous measurements (Zhao et al. 2007), equimolar DOPC/DSPC consists of a tilted gel phase coexisting with a fluid phase. However, for this mixture, the gel/fluid boundary is thought to be even closer to pure DSPC. We therefore suggest that $X_{2 \text { phase }}<X_{0 \text { tilt }}$, so that addition of DOPC to DSPC causes phase separation before it eliminates the average chain tilt, whereas in DPPC phase separation requires more DOPC in the gel phase and this additional DOPC eliminates the average chain tilt. 

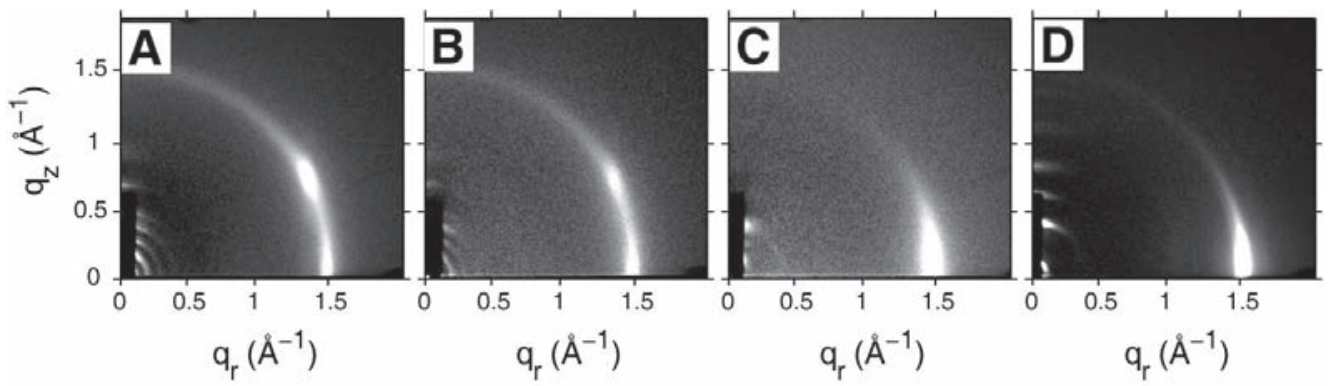

Figure 4. Linear grayscale CCD images as described in Fig. 1. A. gel phase $\left(\mathrm{L}_{\beta^{\prime}}\right)$ DPPC $(D=61.8 \AA)$; B. 0.02/0.98 Chol/DPPC $(D=$ $63.1 \AA)$; C. $0.07 / 0.93 \mathrm{Chol} / \mathrm{DPPC}(D=82.9 \AA)$; D. DPPE $(D=60.4 \AA)$, all at $25^{\circ} \mathrm{C}$. All rotating anode data.

Small amounts of Chol cause dramatic changes in chain packing and orientation

Fig. 4 shows that small concentrations of Chol have a drastic effect on chain packing and orientation in DPPC bilayers at $25^{\circ} \mathrm{C}$. As previously discussed in connection with Fig. 1A, Fig. 4A reminds us that DPPC is in the tilted $\mathrm{L}_{\beta}$, phase. For a $0.02 / 0.98$ Chol/DPPC mixture, two clearly separated WAXS peaks are still observable as seen in Fig. 4B; however, for 0.07/0.93 Chol/DPPC Fig. 4C shows only an equatorial concentration of intensity, suggesting that the average tilt of the chains is zero. Fig. 4D shows for comparison oriented WAXS data for DPPE, which has a single equatorial peak (at $d=4.12 \AA$ ), in agreement with McIntosh (1980) that DPPE is in the untilted $\mathrm{L}_{\beta}$ phase at $25^{\circ} \mathrm{C}$.

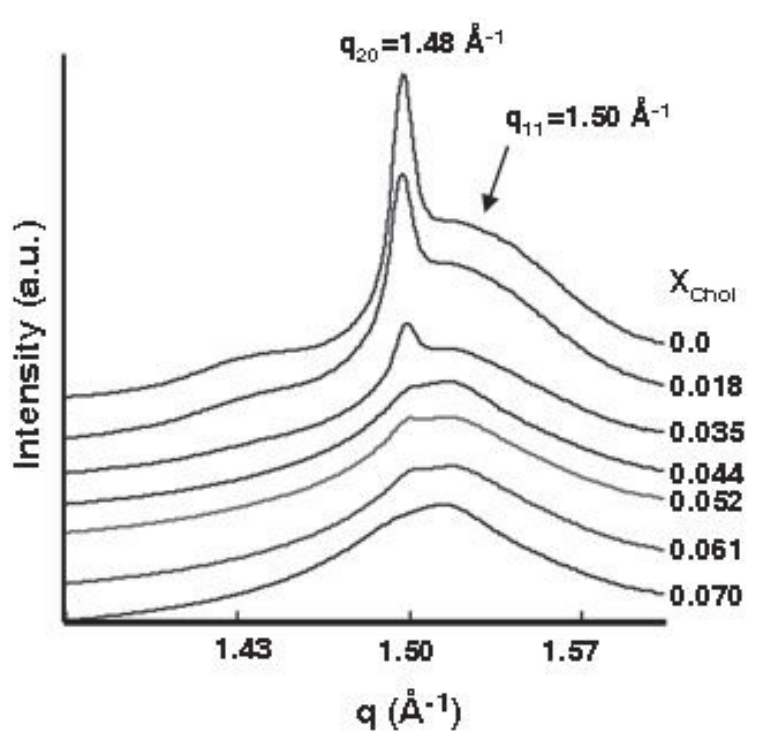

Figure 5. Intensity $I(q)$ plots from unoriented Chol/DPPC MLVs at room temperature $\left(24^{\circ} \mathrm{C}\right)$. Data collected with setup MLV 1 as described in Materials and Methods.
This untilting with addition of Chol is similar to the untilting that occurs when small amounts of DOPC are added to DPPC. In the latter case, the untilting is not associated with the phase coexistence that occurs at higher concentrations of DOPC, so it is also not necessary to associate the untilting of chains at low concentrations of Chol with a two phase coexistence region.

While not as definitive as data from oriented samples, the WAXS data from unoriented MLVs in Fig. 5 also show that the narrow $(2,0)$ peak and broader $(1,1)$ peak, which are characteristic of the $\mathrm{L}_{\beta}$, phase, are gradually replaced by a single broader peak with the addition of Chol. An advantage of MLV data is that the lamellar $D$ spacing may easily be obtained for samples that are always fully hydrated. The $D$ spacing data in Fig. 6 are also consistent with dramatic changes that occur at

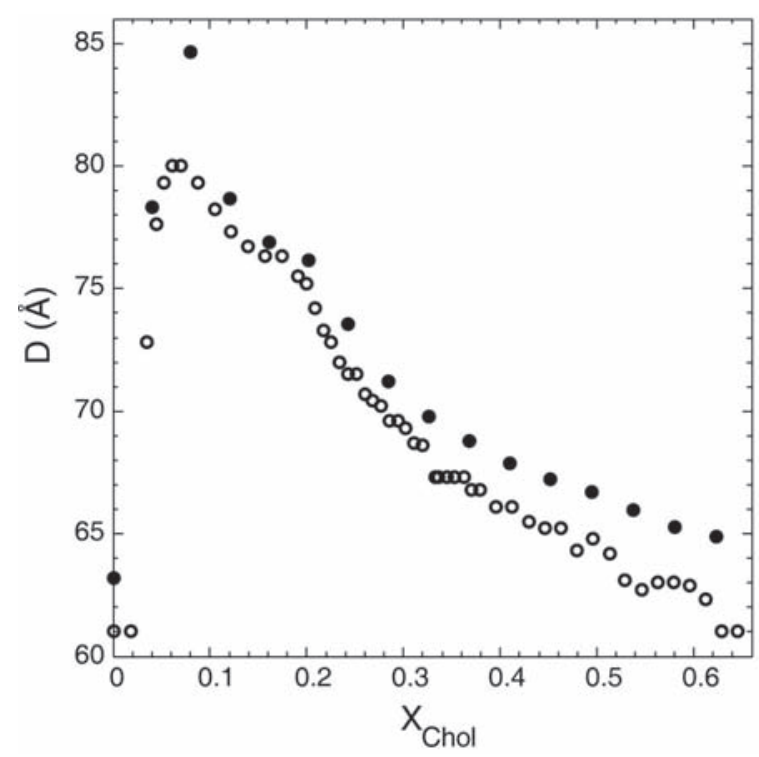

Figure 6. Lamellar repeat $D$ is plotted versus mole fraction Chol for unoriented MLV samples of Chol/DPPC at $24^{\circ} \mathrm{C}$. Filled circles and open circles represent two different data sets. Data collected with setup MLV 1 as described in Materials and Methods. 
low concentrations of Chol. The $20 \AA$ increase in $D$ that occurs for $X_{\text {Chol }}=0.08$ has been attributed to $\sim 7 \AA$ increase in bilayer thickness, which is consistent with the removal of chain tilt, and $\sim 13 \AA$ increase in the water spacing (Ladbrooke et al. 1968). This remarkable increase in water spacing, followed by the more gradual decrease in $D$ at higher concentration, requires major changes in the interactions between bilayers, and the swelling has been attributed to either a decrease in van der Waals attraction between bilayers or an increase in repulsion (Rand et al. 1980). It may also be noted that, while low concentrations of Chol and DOPC both remove DPPC chain tilt, the effect on $D$ is very different; all of the DOPC/DPPC mixtures we studied (both unoriented and oriented samples) had lamellar repeats in the 63-64 A range.

The HWHM of the scattering peak is inversely related to the correlation length, and therefore it reports on the positional ordering in the sample. For the DPPC and DPPE images shown in Fig. 4A and D, the HWHM for an $I(q)$ plot integrated over a $5^{\circ}<\varphi<10^{\circ}$ range was $0.04 \AA^{-1}$. Previous high-resolution WAXS data collected on unoriented MLV samples of gel-phase DPPC indicate that the true $\mathrm{HWHM}_{\text {intrinsic }}$ of the $(2,0)$ peak is $\sim 0.002 \AA^{-1}$ (Sun et al. 1994). For our resolution-limited data $\mathrm{HWHM}_{\text {intrinsic }}$ was estimated as:

$$
\mathrm{HWHM}_{\text {intrinsic }}=\left(\mathrm{HWHM}_{\mathrm{obs}}^{2}-\mathrm{HWHM}_{\mathrm{res}}^{2}\right)^{1 / 2}
$$

where $\mathrm{HWHM}_{\mathrm{obs}}$ is the measured value and $\mathrm{HWHM}_{\text {res }}$ is the resolution limit obtained from our gel phase DPPC $(2,0)$ peak. This gives $\mathrm{HWHM}_{\text {intrinsic }}=0.06 \AA^{-1}$ for $0.07 / 0.93$ Chol/DPPC, which is $\sim 30$ times larger than the non-resolution limited value for pure DPPC. So small amounts of Chol not only cause untilting of the chains but also introduce disorder into the regular packing of the chains in gel-phase DPPC.

The dependence on $\varphi$ (defined in Fig. 1) of the oriented WAXS data gives information about acyl chain orientation. Fig. 7 shows $I(\varphi)$ plots of the WAXS images shown in Fig. 4, which help identify the intensity maximum, from which a tilt angle can be calculated. (Note that intensities at small angles (below $\varphi \sim 5^{\circ}$ ) are decreased greatly due to absorption artifacts (Mills et al. 2008a).) For 0.02/0.98 Chol/DPPC, the relative intensity of the $(1,1)$ peak vs. the $(2,0)$ peak is diminished in comparison with pure DPPC. In addition, the location of the maximum decreases by roughly $1^{\circ}$ compared to pure DPPC, for which the tilt angle has been reported to be $32^{\circ}$ (Tristram-Nagle et al. 1993), indicating that the chains are still tilted for at least some part of the sample at nearly the same angle as in pure DPPC. Interestingly, for $0.035 / 0.965 \mathrm{Chol} / \mathrm{DPPC}$, the chain tilt might be changing to an $\mathrm{L}_{\beta}$ F state with chains tilting between nearest neighbors (Smith et al. 1990). For 0.07/0.93 Chol/DPPC, there is no obvious maximum at a nonzero $\varphi$ value, indicating that no part of the sample consists of well-correlated, tilted chains.

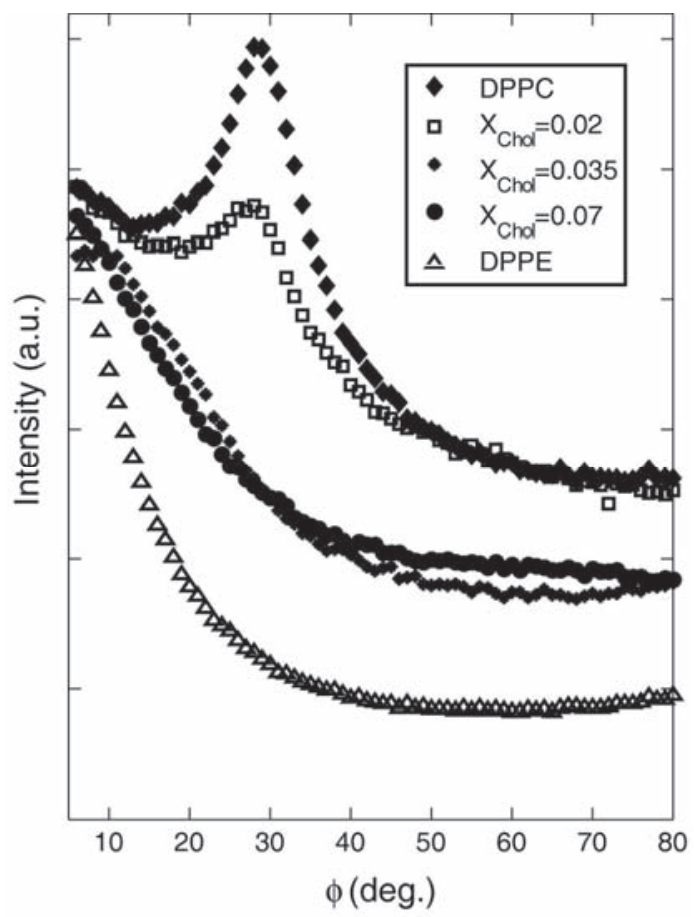

Figure 7. $I(\varphi)$ plots (intensities integrated over a $q=0.8$ to $1.8 \AA^{-1}$ range) for the images shown in Fig. 4, plus a sample with $X_{\mathrm{Chol}}=$ $0.035(D=77.8 \AA)$.

The effect of Chol on gel phase lipids has been reported using X-ray scattering before (Ladbrooke et al. 1968; McIntosh 1978; Rand et al. 1980; Hui and He 1983; Needham et al. 1988; Lemmich et al. 1997). It was concluded, as we do, that the large increase in lamellar repeat (Ladbrooke et al. 1968; McIntosh 1978) and the disappearance (Hui and He 1983) of the characteristic wide angle pattern in unoriented wide angle scattering data (see Fig. 5) indicates a loss of chain tilt with the addition of Chol.

The language of "chain tilt" is clear when referring to well-ordered gel phases, but it is confusing when referring to disordered phases. As an extreme example, in the $\mathrm{L}_{\mathrm{d}}$ phase, the average angle $\beta$ at which the chains tilt with respect to the membrane normal is zero (i.e. the director points along the membrane normal), but the chains are orientationally disordered, and so $\langle|\beta|>\neq 0$. Our oriented WAXS data indicate that the average tilt angle, $\langle\beta>$, in $0.07 / 0.93 \mathrm{Chol} / \mathrm{DPPC}$ is zero, as in pure $\mathrm{L}_{\beta}$ phase DPPE. However, the HWHM is broader for $0.07 / 0.93 \mathrm{Chol} / \mathrm{DPPC}$ compared with DPPE, in agreement with previous studies of Chol/DMPC (below $T_{m}$ for DMPC), which described these bilayers as a "very defective lattice" (Hui and He 1983) or "weakly solid" (Needham et al. 1988). In addition to showing that $0.07 / 0.93 \mathrm{Chol} / \mathrm{DPPC}$ is more positionally disordered than DPPE, the oriented WAXS data show that $0.07 / 0.93 \mathrm{Chol} / \mathrm{DPPC}$ is more orientationally disordered, based on the broader $I(\varphi)$ distribution (see 

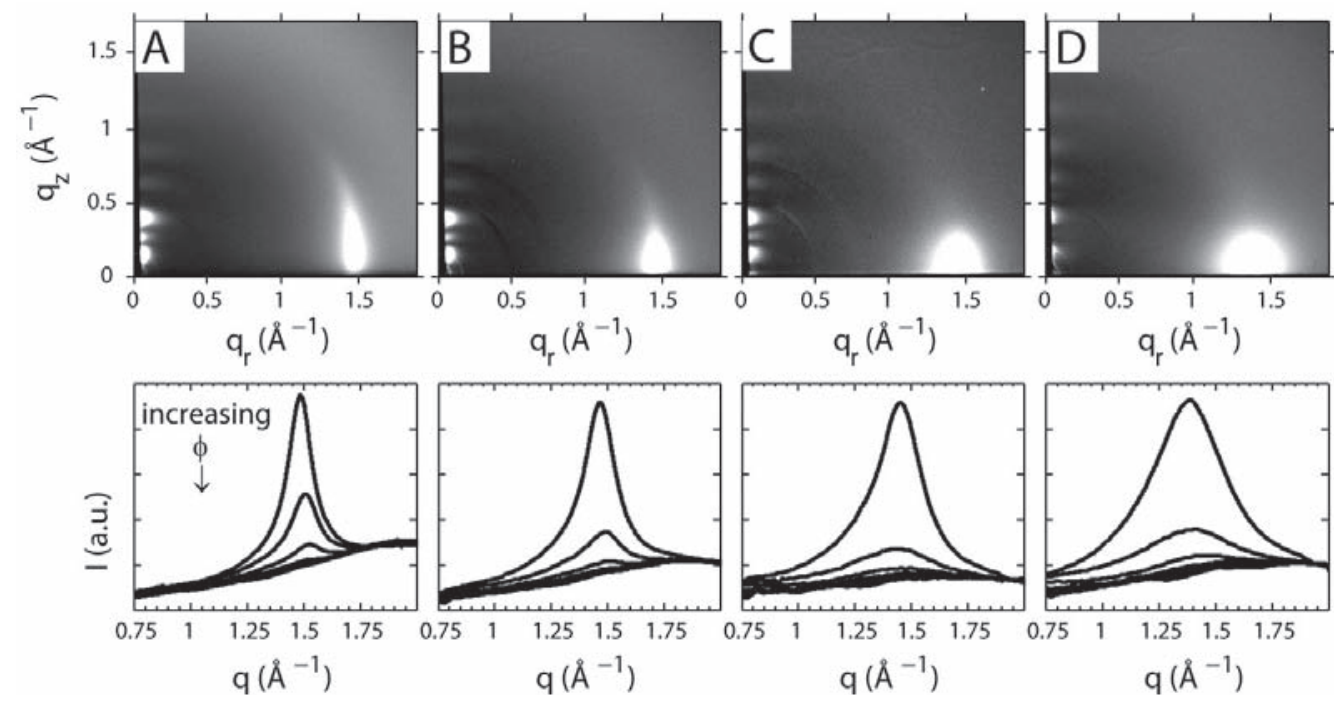

Figure 8. Two dimensional CCD images for: A. 0.10/0.90 Chol/DPPC ( $D=77.5 \AA$; compared with $D=80.5 \AA$ for MLV 3); B. $0.15 / 0.85$ Chol/DPPC ( $D=75.9 \AA \AA$; compared with $D=78.4 \AA$ for MLV 3); C. $0.25 / 0.75$ Chol/DPPC $(D=68.2 \AA$; compared with $D=73.5 \AA ̊$ for MLV 3); D. 0.40/0.60 Chol/DPPC ( $D=66.7 \AA \AA$; compared with $D=67.3 \AA$ for MLV 3) (all at $\left.25^{\circ} \mathrm{C}\right)$. The bottom row shows the corresponding $I(q)$ plots integrated over $\varphi$ ranges of $5-15^{\circ}, 15-25^{\circ}, \ldots, 75-85^{\circ}$. Data collection and processing are described in detail in a previous paper (Mills et al. 2008a). Uncertainties in measured intensities are $1 \%$ of the maximum intensity. All synchrotron data.
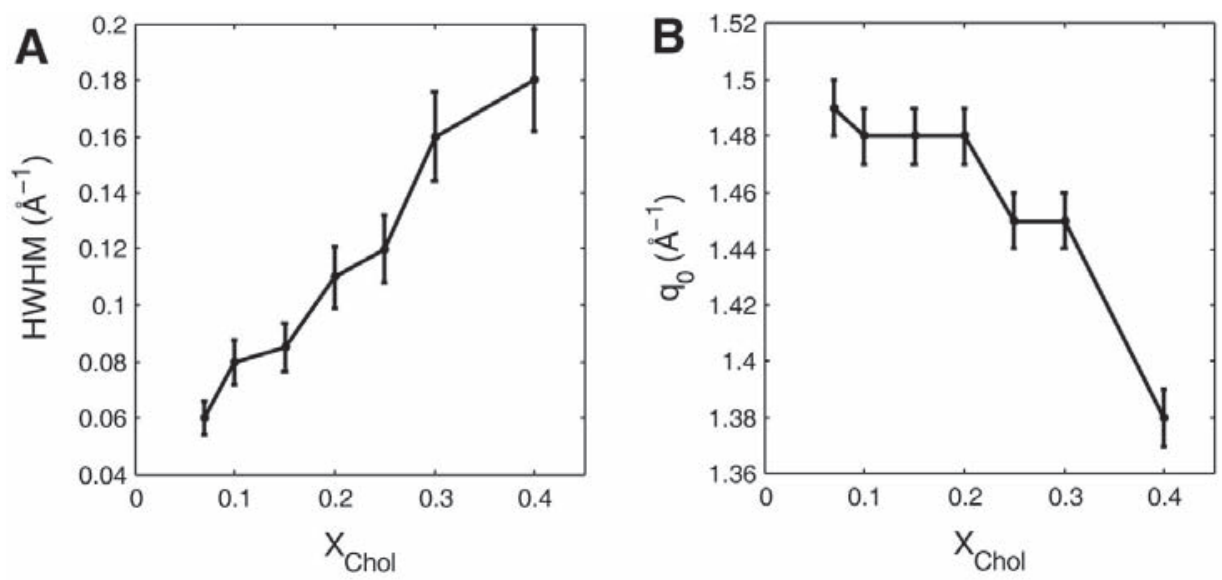

Figure 9. HWHM (A) and peak maximum position, $q_{0}(\mathrm{~B})$, as a function of mole fraction Chol for Chol/DPPC I(q) plots (intensity integrated over a $\varphi=5$ to $10^{\circ}$ range).

Fig. 7), indicating a broader distribution of chain tilt angles. So, although the chain orientation director may point along the membrane normal (i.e. $\langle\beta\rangle=0$ ) for both these samples, $<|\beta|>$ appears to be greater for 0.07/0.93 Chol/DPPC.

Gradual changes in chain packing observed at moderate Chol concentrations $\left(X_{\text {Chol }}=0.07\right.$ to 0.40)

In comparison with the dramatic change in DPPC chain packing and orientation which occurs for $X_{\text {Chol }}$ between 0 and 0.07 (seen in Fig. 4), Fig. 8 shows that the changes in the WAXS data are more gradual for higher Chol concentrations. In agreement with previous reports (Vist and Davis 1990; Scheidt et al. 2005), for $X_{\mathrm{Chol}}$ up to 0.40, the lipid maintains a high degree of orientational order, as is evident by the concentration of the scattering intensity near $\varphi=0$ for all the Chol concentrations shown in Fig. 8. However, Fig. 9A shows that $\mathrm{HWHM}_{\text {intrinsic }}$ increases steadily for $X_{\mathrm{Chol}}=$ 0.07 to 0.40 , indicating that Chol gradually decreases the positional ordering. The $\mathrm{HWHM}_{\text {intrisic }}$ of $0.18 \AA^{-1}$ in the 
$I(q)$ plot falls in the range we have observed $\left(0.14-0.18 \AA^{-1}\right)$ for fluid phases with varying degrees of Chol (Mills et al. 2008a). Another relevant x-ray parameter to track is the position $q_{0}$ of the peak maximum. Fig. $9 \mathrm{~B}$ shows that Chol decreases $q_{0}$, as has been reported previously for a number of phospholipids (Hui and He 1983; Finean 1990). Although this might suggest an increase in chain separation, one must keep in mind that the total scattering includes Chol-lipid and Chol-Chol scattering.

Fig. 10 shows that increasing temperature also causes an increase in the HWHM. In contrast to the gradual change in the HWHM as a function of Chol, the increase in the HWHM occurs in a narrow range of temperature in 0.15/0.85 Chol/DPPC, as one expects due to the main chain melting which necessarily involves positionally disordering. However, for $0.40 / 0.60 \mathrm{Chol} / \mathrm{DPPC}$, there is no significant jump; at $25^{\circ} \mathrm{C}$ the $\mathrm{HWHM}$ is $0.18 \AA^{-1}$ and at $45^{\circ} \mathrm{C}$ it is $0.16 \AA^{-1}$ (Mills et al. 2008a). The broadening and gradual disappearance of the jump in the HWHM vs. temperature plot has been documented previously for Chol/DMPC mixtures (Hui and He 1983). The gradual disappearance of a thermal transition as more Chol is added has also been observed in many other measurements: DSC (Mabrey et al. 1978), NMR first moment (Jacobs and Oldfield 1979; Vist and Davis 1990; Scheidt et al. 2005), translational diffusion (Rubenstein et al. 1979), and volume (Melchior et al. 1980).

\section{Interpretation of $x$-ray data regarding phase coexistence}

As previously formulated (Mills et al. 2008b), there are several x-ray criteria for phase coexistence. These criteria include: 1 - the observation of two lamellar $D$ spacings and 2 - the observation of two WAXS $d$ spacings. Either of these traditional criteria is sufficient but not necessary for phase coexistence. As already presented, in the equimolar mixture of DOPC and DPPC at $25^{\circ} \mathrm{C}$ for which GUV images clearly show coexisting gel and fluid domains (Veatch and Keller 2003), we only observed a single lamellar repeat, so $\mathrm{x}$-ray criterion 1 is not necessary for phase coexistence. Reasons for the non-necessity of criterion 1 are that a phaseseparated sample can have a single lamellar repeat either because the two phases have similar $D$ spacings or else because the domains do not align across bilayers (Rand et al. 1980; Mills et al. 2008b). For the same equimolar DOPC/DPPC sample, the oriented WAXS data clearly showed a sharp peak (gel) and broad peak $\left(\mathrm{L}_{\mathrm{d}}\right)$, so $\mathrm{x}$-ray criterion 2 is sufficient to establish phase coexistence. However, this criterion may also not be necessary; for example, in the case of $\mathrm{L}_{\mathrm{d}} / \mathrm{L}_{\mathrm{o}}$ phase coexistence, only a single WAXS peak may be observed because the scattering from both phases is diffuse and overlapping. Because these two traditional x-ray criteria can give false negative diagnoses of phase coexistence, we previously developed a third $\mathrm{x}$-ray criterion involving fitting

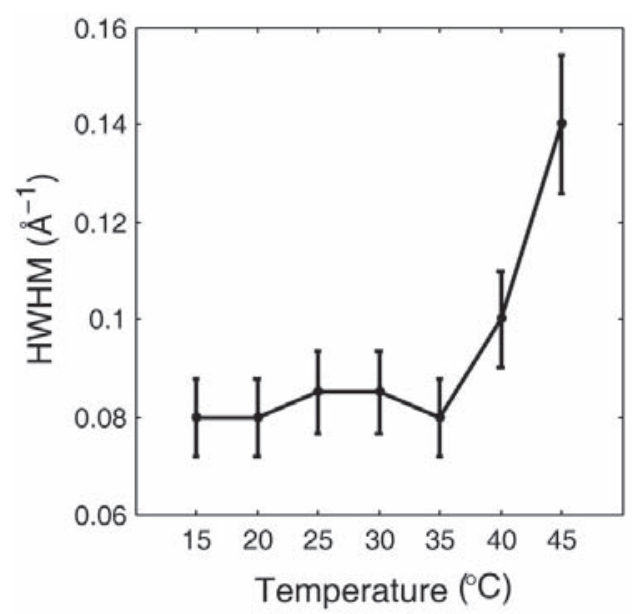

Figure 10. HWHM of the $I(q)$ plot (intensity integrated over a $\varphi=5$ to $10^{\circ}$ range) is plotted versus temperature for $0.15 / 0.85$ Chol/DPPC.

of $I(\varphi)$ data (Mills et al. 2008b). Unfortunately, that third criterion is only applicable to liquid-liquid coexistence and should not be used on gel phases. In this section, we discuss our data and the literature with regard to the two traditional $\mathrm{x}$-ray criteria and we introduce a fourth criterion.

Previous studies (Rand et al. 1980; Meyer et al. 1997) have reported two lamellar repeats (criterion 1) in Chol/ DPPC mixtures, but for $X_{\mathrm{Chol}}=0.03$ to 0.10 , much lower than the Vist and Davis coexistence region. In Chol/DPPC mixtures, we observed only a single lamellar repeat in both oriented and fully hydrated MLV samples. Engelman and Rothman (1972) reported two WAXS peaks in Chol/DPPC mixtures in the range of $X_{\mathrm{Chol}}=0.20$ to 0.33 . In contrast, in oriented WAXS Chol/DPPC samples, we only observed a single WAXS peak. Therefore, in our hands, neither of the two traditional criteria was sufficient to predict phase coexistence. Together with the observation of uniform fluorescence in microscopy images of GUVs (Feigenson and Buboltz 2001; Veatch and Keller 2005), one might conclude that there is no phase coexistence in Chol/DPPC mixtures at $25^{\circ} \mathrm{C}$. However, neither of the traditional $\mathrm{x}$-ray criteria is necessary, and the fluorescence microscopy can only conclude that phase coexistence would occur on length scales smaller than microns.

As a possible reason for not observing two wide angle peaks, Hui and He (1983) noted that their samples were measured immediately after preparation, which may not allow sufficient time for a gel phase to form. However, we observed no change in the WAXS pattern for a $0.15 / 0.85$ Chol/DPPC sample after 3 days at high humidity, indicating that insufficient equilibration time is not the reason we fail to observe two WAXS peaks in a region where other measurements suggest gel/fluid phase separation. Therefore, 

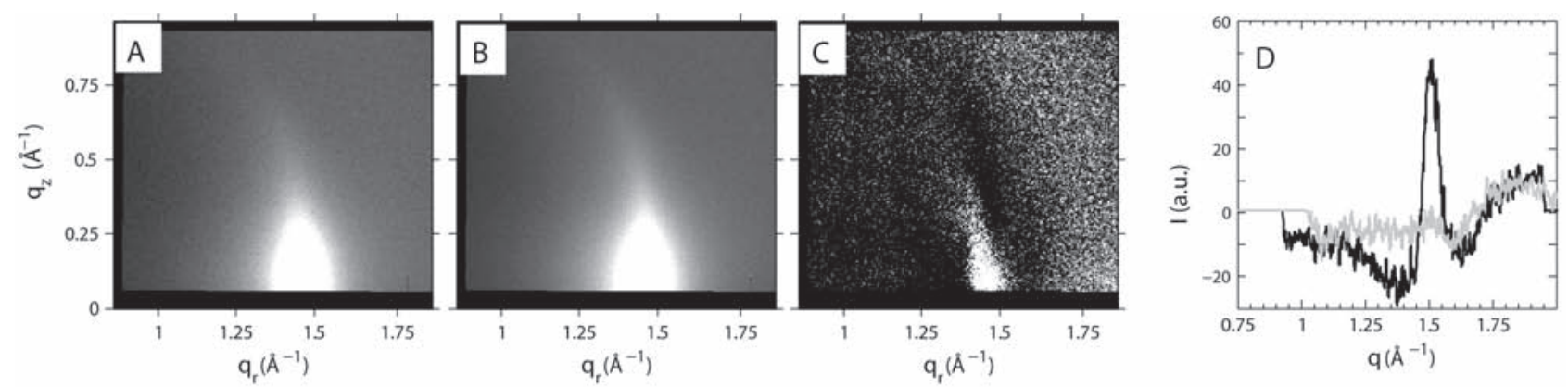

Figure 11. A. Two dimensional CCD image for $0.15 / 0.85 \mathrm{Chol} / \mathrm{DPPC}$ at $25^{\circ} \mathrm{C}$ (same image as Fig. $8 \mathrm{~B}$, but narrower $q$ range). B. Least squares fit of a linear combination of images from samples of 0.10/0.90 Chol/DPPC and 0.25/0.75 Chol/DPPC (images were shown in Fig. $8 \mathrm{~A}$ and $\mathrm{C}$ ) to the data in (A). C. Image shown in (B) subtracted from the image shown in (A). The grayscale for (C) is expanded by a factor of 17 with respect to the scales in (A) and (B). D. The differences in intensity $\Delta \mathrm{I}$ in (C) are shown as a function of total $\mathrm{q}$ for data in the sector $5-10^{\circ}$ (black line) and data in the sector $25-30^{\circ}$ (light gray line). The gradually increasing $\Delta I$ for larger $q$ is the same for different angular sectors, indicating that it is isotropic and due to different amounts of water in the different samples.

our WAXS data are inconsistent with a fluid coexisting with a highly ordered gel phase. However, as pointed out previously (Clarke et al. 2006), for a "disordered gel" phase in coexistence with an $\mathrm{L}_{\mathrm{O}}$ phase, one might expect the WAXS data to overlap, as in the case of diffuse WAXS data from two coexisting liquid phases.

Instead of simply looking for a narrow and broad peak in the WAXS pattern, we have devised a different test whether the Vist and Davis phase diagram could be consistent with our WAXS data. If there is phase separation in the reported range of $X_{\mathrm{Chol}}=0.10$ to 0.25 (within the Vist and Davis range with error), then our $0.15 / 0.85 \mathrm{Chol} / \mathrm{DPPC}$ oriented WAXS image should be the same as the sum of $2 / 3$ of our $0.10 / 0.90 \mathrm{Chol} / \mathrm{DPPC}$ and $1 / 3$ of our $0.25 / 0.75 \mathrm{Chol} / \mathrm{DPPC}$ WAXS images, if we assume that the amount of material is equal in the two samples. Even if the assumption is incorrect, as it generally is in $\mathrm{x}$-ray studies, a mass weighted linear combination is still required to be the same as the 0.15/0.85 Chol/DPPC image; this application of the lever rule to WAXS images establishes a new criterion 4. Fig. 11A shows the original image and Fig. 11B shows a fit of a linear combination of the latter two images to a portion of the former image which contains most of the WAXS intensity that is free of artifacts due to absorption and LAXS/lamellar scattering. The fitted image looks similar to the directly measured one. However, this comparison does not focus on the differences. Such differences would be expected to be small for states with similar properties. Nevertheless, any statistically significant small differences from a strict linear combination suffices to prove that phase coexistence does not exist over the range of the three concentrations. The logical equivalent is that criterion 4 is necessary for phase coexistence in the concentration region in which it is applied. Fig. 11C shows the differences between the best fitted linear combination and the data for the $0.15 / 0.85$ $\mathrm{Chol} / \mathrm{DPPC}$ sample. There is a large, statistically significant, region consisting of 2500 pixels centered at $q_{\mathrm{r}}=1.5 \AA^{-1}, q_{\mathrm{z}}=$ $0.1 \AA^{-1}$ where all the differences are positive surrounded by two other large regions where all the differences are negative. This is quantified in Fig. 11D which shows that the differences are statistically significant even in a one dimensional plot. Other regions where there is less WAXS scattering have uniform differences. While this kind of analysis must be repeated on additional data sets, including controls on samples like DOPC/DPPC where there is definitely two phase coexistence, this is the only type of $\mathrm{x}$-ray result that can show that phase coexistence does not occur in a region such as $X_{\mathrm{Chol}}=0.10$ to 0.25 in DPPC at $25^{\circ} \mathrm{C}$. Unlike criteria 1 and 2 which are sufficient criteria for phase coexistence, this criterion 4 is sufficient to establish non-coexistence of phases in the concentration range to which it is applied.

\section{Summary}

We have critically examined $x$-ray evidence pertinent to the phase behavior of DOPC/DPPC and Chol/DPPC mixtures. Our WAXS data for DOPC/DPPC support the two phase coexistence observed by fluorescence microscopy. Our data for Chol/DPPC do not support the traditional $\mathrm{x}$-ray criterion 1 (two coexisting LAXS D spacings) or criterion 2 (two coexisting WAXS $d$ spacings) for phase coexistence, but we have emphasized that the observance of only one LAXS $D$ spacing and of only one apparent WAXS $d$ spacing, while necessary criteria for a single phase, are not sufficient to rule out phase coexistence. However, the greatest change in our WAXS data takes place in a range of $X_{\mathrm{Chol}}=0$ to 0.07 , where the system transforms from a tilted gel phase with consider- 
able short range order to a more disordered, untilted solid state. For $X_{\text {Chol }}>0.07$ the changes in the WAXS data are much smaller. In contrast, phase diagrams in the literature predict phase coexistence in Chol concentration ranges with a minimum value greater than $X_{\mathrm{Chol}}=0.07$. We have introduced a new criterion 4 that involves global comparison of WAXS data at three concentrations. At this time, our result using criterion 4 implies that phase coexistence does not encompass the concentration range from $X_{\mathrm{Chol}}=0.10$ to 0.25 in $\mathrm{Chol} / \mathrm{DPPC}$ mixtures at $25^{\circ} \mathrm{C}$, and this result is consistent with the traditional, but inconclusive, criteria 1 and 2 .

Acknowledgements. J. F. Nagle appreciates being asked to contribute a paper to honor Prof. Pavol Balgavy, and to partially repay him for sending his former student Dr. Norbert Kučerka to be a postdoc in our laboratory. We acknowledge Prof. Stephanie Tristram-Nagle for help with experiments and analysis. T. T. Mills thanks Dr. Gilman Toombes for discussions and Dr. Mark Tate for help with those rotating anode experiments on unoriented samples that were performed at the Cornell facility, supported by Department of Energy award DE-FG02-97ER62443 to Prof. Sol Gruner. We acknowledge beamline support from Dr. Detlef-M. Smilgies and use of beam time at CHESS which is supported by the National Science Foundation and the National Institutes of Health/National Institute of General Medical Sciences under NSF award DMR-0225180. This work was supported by grants from the National Institutes of Health (GM 44976) to J. F. Nagle and the National Science Foundation (MCB-0315330) to G. W. Feigenson. T. T. Mills was supported in part by NIH research award (1-T32-GM08267).

\section{References}

Almeida P. F. F., Vaz W. L. C., Thompson T. E. (1993): Percolation and diffusion in three-component lipid bilayers: effect of cholesterol on an equimolar mixture of two phosphatidylcholines. Biophys. J. 64, 399-412; doi:10.1016/S00063495(93)81381-X

Bloom M., Thewalt J. L. (1995): Time and distance scales of membrane domain organization. Mol. Membr. Biol. 12, 9-13; doi:10.3109/09687689509038489

Chen L., Yu Z. W., Quinn P. J. (2007): The partition of cholesterol between ordered and fluid bilayers of phosphatidylcholine: a synchrotron X-ray diffraction study. Biochim. Biophys. Acta 1768, 2873-2881; doi:10.1016/j.bbamem.2007.07.023

Clarke J. A., Heron A. J., Seddon J. M., Law R. V. (2006): The diversity of the liquid ordered (Lo) phase of phosphatidylcholine/cholesterol membranes: a variable temperature multinuclear solid-state NMR and x-ray diffraction study. Biophys. J. 90, 2383-2393; doi:10.1529/ biophysj.104.056499

Edholm O., Nagle J. F. (2005): Areas of molecules in membranes consisting of mixtures. Biophys. J. 89, 1827-1832; doi:10.1529/biophysj.105.064329

Engelman D. M., Rothman J. E. (1972): The planar organization of lecithin-cholesterol bilayers. J. Biol. Chem. 247, 3694-3697
Feigenson G. W., Buboltz J. T. (2001): Ternary phase diagram of dipalmitoyl-PC/dilauroyl-PC/cholesterol: nanoscopic domain formation driven by cholesterol. Biophys. J. 80, 2775-2788; doi:10.1016/S0006-3495(01)76245-5

Finean J. B. (1990): Interaction between cholesterol and phospholipid in hydrated bilayers. Chem. Phys. Lipids 54, 147-156; doi:10.1016/0009-3084(90)90008-F

Finegold L. (1993): Cholesterol in Membrane Models. CRC Press, Boca Raton

Gallová J., Uhríková D., Islamov A., Kuklin A., Balgavý P. (2004): Effect of cholesterol on the bilayer thickness in unilamellar extruded DLPC and DOPC liposomes: SANS contrast variation study. Gen. Physiol. Biophys. 23, $113-128$

Greenwood A. I., Tristram-Nagle S., Nagle J. F. (2006): Partial molecular volumes of lipids and cholesterol. Chem. Phys. Lipids 143, 1-10; doi:10.1016/j.chemphyslip.2006.04.002

Heerklotz H., Tsamaloukas A. (2006): Gradual change or phase transition: characterizing fluid lipid-cholesterol membranes on the basis of thermal volume changes. Biophys. J. 91, 600-607; doi:10.1529/biophysj.106.082669

Hsueh Y.-W. Gilbert K., Trandum C., Zuckermann M., Thewalt J. (2005): The effect of ergosterol on dipalmitoylphosphatidylcholine bilayers: a deuterium NMR and calorimetric study. Biophys. J. 88, 1799-1808; doi:10.1529/ biophysj.104.051375

Huang J. Y., Feigenson G. W. (1999): A microscopic interaction model of maximum solubility of cholesterol in lipid bilayers. Biophys. J. 76, 2142-2157; doi:10.1016/S00063495(99)77369-8

Huang J. Y., Buboltz J. T., Feigenson G. W. (1999): Maximum solubility of cholesterol in phosphatidylcholine and phosphatidylethanolamine bilayers. Biochim. Biophys. Acta 1417, 89-100; doi:10.1016/S0005-2736(98)00260-0

Huang T. H., Lee C. W., Das Gupta S. K., Blume A., Griffin R. G. (1993): A 13C and 2H nuclear magnetic resonance study of phosphatidylcholine/cholesterol interactions: characterization of liquid-gel phases. Biochemistry 32, 13277-13287; doi:10.1021/bi00211a041

Hui S. W., He N. B. (1983): Molecular organization in cholesterol-lecithin bilayers by $\mathrm{x}$-ray and electron diffraction measurements. Biochemistry 22, 1159-1164; doi:10.1021/ bi00274a026

Ipsen J. H., Karlström G., Mouritsen O. G., Wennerström H., Zuckermann M. J. (1987): Phase equilibria in the phosphatidylcholine-cholesterol system. Biochim. Biophys. Acta 905, 162-172; doi:10.1016/0005-2736(87)90020-4

Jacobs R., Oldfield E. (1979): Deuterium nuclear magnetic resonance investigation of dimyristoyllecithin-dipalmitoyllecithin and dimyristoyllecithin-cholesterol mixtures. Biochemistry 18, 3280-3285; doi:10.1021/bi00582a013

Janiak M. J., Small D. M., Shipley G. G. (1976): Nature of the thermal pretransition of synthetic phospholipids: dimyristoyland dipalmitoyllecithin. Biochemistry 15, 4575-4580; doi:10.1021/bi00666a005

Karmakar S., Raghunathan V. A. (2003): Cholesterol-induced modulated phase in phospholipid membranes. Phys. Rev. Lett. 91, 098102; doi:10.1103/PhysRevLett.91.098102 
Karmakar S., Sarangi B. R., Raghunathan V. A. (2006): Phase behaviour of lipid-cholesterol membranes. Solid State Commun. 139, 630-634; doi:10.1016/j.ssc.2006.05.045

Kingsley P. B., Feigenson G. W. (1979): Synthesis of a perdeuterated phospholipid: 1,2-dimyristoyl-sn-glycero-3phosphocholine-d72. Chem. Phys. Lipids 24, 135-147; doi:10.1016/0009-3084(79)90083-5

Krivanek R., Okoro L., Winter R. (2008): Effect of cholesterol and ergosterol on the compressibility and volume fluctuations of phospholipid-sterol bilayers in the critical point region: a molecular acoustic and calorimetric study. Biophys. J. 94, 3538-3548; doi:10.1529/biophysj.107.122549

Kučerka N., Liu Y. F., Chu N. J., Petrache H. I., Tristram-Nagle S., Nagle J. F. (2005): Structure of fully hydrated fluid phase DMPC and DLPC lipid bilayers using $\mathrm{x}$-ray scattering from oriented multilamellar arrays and from unilamellar vesicles. Biophys. J. 88, 2626-2637; doi:10.1529/ biophysj.104.056606

Kučerka N., Perlmutter J. D., Pan J., Tristram-Nagle S., Katsaras J., Sachs J. N. (2008): The effect of cholesterol on short- and long-chain monounsaturated lipid bilayers as determined by molecular dynamics simulations and x-ray scattering. Biophys. J. 95, 2792-2805; doi:10.1529/ biophysj.107.122465

Ladbrooke B. D., Williams R. M., Chapman D. (1968): Studies on lecithin-cholesterol-water interactions by differential scanning calorimetry and $\mathrm{x}$-ray diffraction. Biochim. Biophys. Acta 150, 333-340; doi:10.1016/00052736(68)90132-6

Lemmich J., Mortensen K., Ipsen J. H., Hønger T., Bauer R., Mouritsen O. G. (1997): The effect of cholesterol in small amounts on lipid-bilayer softness in the region of the main phase transition. Eur. Biophys. J. 25, 293-304; doi: $10.1007 /$ s002490050041

Lentz B. R., Barenholz Y., Thompson T. E. (1976): Fluorescence depolarization studies of phase transitions and fluidity in phospholipid bilayers. 2. Two-component phosphatidylcholine liposomes. Biochemistry 15, 4529-4537; doi:10.1021/bi00665a030

Levine Y. K., Wilkins M. H. F. (1971): Structure of oriented lipid bilayers. Nature New Biol. 230, 69-72

Li L., Cheng J.-X. (2006): Coexisting stripe- and patch-shaped domains in giant unilamellar vesicles. Biochemistry 45, 11819-11826; doi:10.1021/bi060808h

Lindblom G., Johansson L. B.-A., Arvidson G. (1981): Effect of cholesterol in membranes. Pulsed nuclear magnetic resonance measurements of lipid lateral diffusion. Biochemistry 20, 2204-2207; doi:10.1021/bi00511a020

Mabrey S., Mateo P. L., Sturtevant J. M. (1978): High-sensitivity scanning calorimetric study of mixtures of cholesterol with dimyristoyl- and dipalmitoylphosphatidylcholines. Biochemistry 17, 2464-2468; doi:10.1021/bi00605a034

Mathai J. C., Tristram-Nagle S., Nagle J. F., Zeidel M. L. (2008): Structural determinants of water permeability through the lipid membrane. J. Gen. Physiol. 131, 69-76; doi:10.1085/jgp.200709848

McConnell H., Radhakrishnan A. (2006): Theory of the deuterium NMR of sterol-phospholipid membranes. Proc.
Natl. Acad. Sci. U.S.A. 103, 1184-1189; doi:10.1073/ pnas. 0510514103

McIntosh T. J. (1978): The effect of cholesterol on the structure of phosphatidylcholine bilayers. Biochim. Biophys. Acta 513, 43-58; doi:10.1016/0005-2736(78)90110-4

McIntosh T. J. (1980): Differences in hydrocarbon chain tilt between hydrated phosphatidylethanolamine and phosphatidylcholine bilayers: a molecular packing model. Biophys. J. 29, 237-246; doi:10.1016/S00063495(80)85128-9

McMullen T. P., McElhaney R. N. (1995): New aspects of the interaction of cholesterol with dipalmitoylphosphatidylcholine bilayers as revealed by high-sensitivity differential scanning calorimetry. Biochim. Biophys. Acta 1234, 90-98; doi:10.1016/0005-2736(94)00266-R

Melchior D. L., Scavitto F. J., Steim J. M. (1980): Dilatometry of dipalmitoyllecithin-cholesterol bilayers. Biochemistry 19, 4828-4834; doi:10.1021/bi00562a018

Mermin N. D., Wagner H. (1966): Absence of ferromagnetism or antiferromagnetism in one- or two-dimensional isotropic Heisenberg models. Physiol. Rev. Lett. 17, 1133-1136; doi:10.1103/PhysRevLett.17.1133

Mermin N. D. (1968): Crystalline order in two dimensions. Physiol. Rev. 176, 250-254; doi:10.1103/PhysRev.176.250

Meyer H. W., Semmler K., Quinn P. J. (1997): The effect of sterols on structures formed in the gel/subgel phase state of dipalmitoylphosphatidylcholine bilayers. Mol. Membr. Biol. 14, 187-193; doi:10.3109/09687689709048181

Mills T. T., Toombes G. E. S., Tristram-Nagle S., Smilgies D.-M., Feigenson G. W., Nagle J. F. (2008a): Order parameters and areas in fluid-phase oriented lipid membranes using wide angle $x$-ray scattering. Biophys. J. 95, 669-681; doi:10.1529/biophysj.107.127845

Mills T. T., Tristram-Nagle S., Heberle F. A., Morales N. F., Zhao J., Wu J., Toombes G. E. S., Nagle J. F., Feigenson G. W. (2008b): Liquid-liquid domains in bilayers detected by wide angle x-ray scattering. Biophys. J. 95, 682-690; doi:10.1529/biophysj.107.127910

Needham D., McIntosh T. J., Evans E. (1988): Thermomechanical and transition properties of dimyristoylphosphatidylcholine/cholesterol bilayers. Biochemistry 27, 4668-4673; doi:10.1021/bi00413a013

Petrache H. I., Harries D., Parsegian V. A. (2005): Alteration of lipid membrane rigidity by cholesterol and its metabolic precursors. Macromol. Symp. 219, 39-50; doi:10.1002/ masy.200550105

Rand R. P., Parsegian V. A., Henry J. A., Lis L. J., McAlister M. (1980): The effect of cholesterol on measured interaction and compressibility of dipalmitoylphosphatidylcholine bilayers. Can. J. Biochem. 58, 959-968

Richter F., Rapp G., Finegold L. (2001): Miscibility gap in fluid dimyristoylphosphatidylcholine: cholesterol as "seen" by x-rays. Phys. Rev. E 63, 051914; doi:10.1103/ PhysRevE.63.051914

Rubenstein J. L. R., Smith B. A., McConnell H. M. (1979): Lateral diffusion in binary mixtures of cholesterol and phosphatidylcholines. Proc. Natl. Acad. Sci. U.S.A. 76, 15-18; doi:10.1073/pnas.76.1.15 
Sankaram M. B., Thompson T. E. (1990): Modulation of phospholipid acyl chain order by cholesterol. A solid-state $2 \mathrm{H}$ nuclear magnetic resonance study. Biochemistry 29, 10676-10684; doi:10.1021/bi00499a015

Scheidt H. A., Huster D., Gawrisch K. (2005): Diffusion of cholesterol and its precursors in lipid membranes studied by $1 \mathrm{H}$ pulsed field gradient magic angle spinning NMR. Biophys. J. 89, 2504-2512; doi:10.1529/ biophysj.105.062018

Smith G. S., Sirota E. B., Safinya C. R., Plano R. J., Clark N. A. (1990): $\mathrm{X}$-ray structural studies of freely suspended ordered hydrated DMPC multimembrane films. J. Chem. Phys. 92, 4519-4529; doi:10.1063/1.457764

Sun W. J., Suter R. M., Knewtson M. A., Worthington C. R., Tristram-Nagle S., Zhang R., Nagle J. F. (1994): Order and disorder in fully hydrated unoriented bilayers of gel phase dipalmitoylphosphatidylcholine. Phys. Rev. E 49, 4665-4676; doi:10.1103/PhysRevE.49.4665

Tardieu A., Luzzati V., Reman F. C. (1973): Structure and polymorphism of the hydrocarbon chains of lipids: a study of lecithin-water phases. J. Mol. Biol. 75, 711-733; doi:10.1016/0022-2836(73)90303-3

Tate M. W., Gruner S. M., Eikenberry E. F. (1997): Coupling format variations in $\mathrm{x}$-ray detectors based on charge coupled devices. Rev. Sci. Instrum. 68, 47-54; doi:10.1063/ 1.1147611

Tristram-Nagle S., Zhang R., Suter R. M., Worthington C. R., Sun W. J., Nagle J. F. (1993): Measurement of chain tilt angle in fully hydrated bilayers of gel phase lecithins. Biophys. J. 64, 1097-1109; doi:10.1016/S00063495(93)81475-9

Tristram-Nagle S., Liu Y. F., Legleiter J., Nagle J. F. (2002): Structure of gel phase DMPC determined by $\mathrm{x}$-ray diffrac- tion. Biophys. J. 83, 3324-3335; doi:10.1016/S00063495(02)75333-2

Tristram-Nagle S. (2007): Preparation of oriented, fully hydrated lipid samples for structure determination using $\mathrm{x}$-ray scattering. In: Methods in Membrane Lipids. (Ed. A. M. Dopico), pp. 63-75, Humana Press, Totowa; doi:10.1385/1-59745-519-9:63

Veatch S. L., Keller S. L. (2003): Separation of liquid phases in giant vesicles of ternary mixtures of phospholipids and cholesterol. Biophys. J. 85, 3074-3083; doi:10.1016/S00063495(03)74726-2

Veatch S. L., Keller S. L. (2005): Seeing spots: complex phase behavior in simple membranes. Biochim. Biophys. Acta 1746, 172-185; doi:10.1016/j.bbamcr.2005.06.010

Veatch S. L., Gawrisch K., Keller S. L. (2006): Closed-loop miscibility gap and quantitative tie-lines in ternary membranes containing diphytanoyl PC. Biophys. J. 90, 4428-4436; doi:10.1529/biophysj.105.080283

Vist M. R., Davis J. H. (1990): Phase equilibria of cholesterol/dipalmitoylphosphatidylcholine mixtures: $2 \mathrm{H}$ nuclear magnetic resonance and differential scanning calorimetry. Biochemistry 29, 451-464; doi:10.1021/bi00454a021

Yeagle P. L. (1985): Cholesterol and the cell membrane. Biochim. Biophys. Acta 822, 267-287

Zhao J., Wu J., Heberle F. A., Mills T. T., Klawitter P., Huang G., Costanza G., Feigenson G. W. (2007): Phase studies of model biomembranes: complex behavior of DSPC/ DOPC/Chol. Biochim. Biophys. Acta 1768, 2764-2776; doi:10.1016/j.bbamem.2007.07.008

Received: October 6, 2008

Final version accepted: February 4, 2009 